\section{Ecological Economics - Ökonomieverträglichkeit einer Stoffpolitik}

Der Begriff der »Ecological Economics« steht für einen bunten Strauß umweltökonomischer Theorieansätze,

- die in besonderer Weise auf die ökologischen Grenzen wirtschaftlicher Entwicklung aufmerksam machen wollen,

- hierbei im Gegensatz zur klassischen Ressourcentheorie weniger die Knappheitsimplikationen einzelner Ressourcen analysieren, sondern die Bedeutung der umfassender definerten Regelungs- oder Stabilisierungsfunktionen globaler oder regionaler Ökosysteme betonen (Versuch einer ganzheitlichen Sicht der ökologischen Gegebenheiten) und

- in beachtlicher Weise von normativen Postulaten, insbesondere der intergenerationellen Verteilungsgerechtigkeit sowie des von anthropogenen Nutzenüberlegungen abgekoppelten Naturschutzes, ausgehen.

Umweltpolitisch schlagen sich viele Überlegungen der so charakterisierten ökologischen Ökonomie in einer radikalen Sicht der Nachhaltigkeitsinterpretation sowie in der Forderung nach einer umfassenden Stoffpolitik nieder. Typisch hierfür sind viele Teile des ersten Schlußberichts der Enquete-Kommission »Schutz des Menschen und der Umwelt (1) sowie stoffpolitische Forderungen bestimmter Vertreter der deutschen ökologischen Ökonomie (etwa E. U. von Weizsäcker, Schmidt-Bleek, M. Jänicke, U.E.Simonis u. a. ). Erste Anzeichen lassen vermuten, daß sich hierüber in den nächsten Jahren eine interessante Ökonomieverträglichkeits-Diskussion entfalten wird (2). Dies soll nachfolgend anhand neuerer stoffpolitischer Forderungen näher verdeutlicht werden.

Die Vertreter einer umfassenden Stoffpolitik greifen Überlegungen auf, die teilweise bereits früher unter dem Stichwort einer neuen »Chemiepolitik « in die umweltpotitische Diskussion eingebracht worden waren, sich damals aber primär auf die Beeinflussung oder Kontrolle chemischer Stoffe unter Sicherheitsaspekten beschränkten, heute aber unter Bezugnahme auf das Leitbild einer nachhaltigen Wirtschaftsentwicklung auf alle Stoffströme ausgedehnt werden. Stoffpolitik wird hierbei als die zielgerichtete Beeinflussung des Stoffflußvolumens und der Stoffflußstruktur der Welt bzw. ihrer Räume (Wirtschaftsräume, Nationen oder Regionen) definiert. Stand bei den ersten Bemühungen um die Einführung einer neuen Stoffpolitik noch die Beeinflussung einzelner Stoffe bzw. der Stoffflußstruktur zwecks Risikominderung für den Menschen und / oder die Ökosysteme im Vordergrund, konzentrieren sich die heutigen Forderungen unter Bezugnahme auf das Leitbild einer nachhaltigen Wirtschaftsentwicklung zumeist auf die Reduktion des Stoffflußvolumens bei gleichzeitig selektiver Einzelstoffpoitik.
Dieser Wandel hat zwei Gründe. Zum einen erwies sich die umfassende Prüfung aller Einzelstoffe über Produktlinien-Analysen, Ökobilanzen usw. wegen der hierbei zu bewältigenden Informationsbeschaffungs- und Bewertungsprobleme als eine kaum zu bewältigende Aufgabe, zum anderen ergab sich aus der Nachhaltigkeitsdiskussion und den Überlegungen der ökologischen Ökonomie eine neue Sichtweise, die auch zu einer neuen Risikobetrachtung führte. Um dies besser zu verstehen, muß man einen kurzen Blick auf die drei gegenwärtig politisch gehandelten Interpretationen des Nachhaltigkeitsziels werfen Diese Interpretationen beziehen sich auf

- das ökologische Ein-Säulen-Konzept

- das sog. Drei-Säulen-Konzept sowie

- das am Vorsorgeprinzip festgemachte Reduktionskonzept.

\section{Ökologisches Ein-Säulen-Konzept}

Das ökologische Ein-Säulen-Konzept orientiert sich an der klassischen Brundtland-Definition. Danach kann man Sustainable Development ganz allgemein als die Gewährleistung einer mehrere Generationen bzw. unterschiedliche Räume betreffenden Entwicklung, die bestimmten Nachhaltigkeitsanforderungen gerecht wird, kennzeichnen. Da man eine positive Entwicklung zumeist mit einer steigenden Wohlfahrt verbindet, ist nachhaltige Entwicklung die Maximierung der gesellschaftlichen Nettowohlfahrt über mehrere Generationen hinweg unter Mindest-Einhaltung bestimmter Nachhaltigkeits-Nebenbedingungen. Eine solche Interpretation erfordert die Lösung mehrerer Operationalisierungsprobleme. Diese betreffen die Begriffe Entwicklung und Nachhaltigkeit, wobei die primären Probleme sicherlich bei der Konkretisierung der Nachhaltigkeitsbedingungen liegen. Der Festlegung der mindestens zu berücksichtigenden Nebenbedingungen kommt entscheidende Bedeutung zu. Restriktiv gesetzte Nebenbedingungen können nämlich den Entwicklungsspielraum gegen Null tendieren lassen.

Die Nachhaltigkeits-Nebenbedingungen beziehen sich auf solche Tatbestände, die für ein längerfristiges Überleben der menschlichen Gesellschaft(en) relevant sind. Eine Entwicklung ist danach nur dann langfristig aufrechterhaltbar und zukunftsfähig, wenn sie bestimmte Risiken, die eine Entwicklungsstetigkeit in Frage stellen kön nen, vermeidet. Es ist das Verdienst der Umweltforschung, darauf aufmerksam gemacht zu haben, daß es bei Vernachlässigung bestimmter Umweltrisiken zu einem »ökologischen Kollaps « kommen kann, der mit seinen »Folgekosten « die gegenwärtige, insbesondere aber künftige Generationen nicht nur direkt bedroht (etwa in Form des Anstiegs umweltbedingter Krankheiten), sondern auch die wirtschaftliche Entwicklung selbst in Frage zu stellen vermag.
Formal läßt sich das letztgenannte Nachhaltigkeitsproblem mittels einer Produktionsfunktion $\mathrm{Yr}=\mathrm{f}(\mathrm{HK}, \mathrm{SK}, \mathrm{OKK}, \mathrm{TF})$ umschreiben (3). Danach hängt die wirtschaftliche Entwicklung (etwa gemessen mit dem Realeinkommen Yr) nicht nur von den klassischen Faktorenbeständen Humankapital HK, Sachkapital SK und dem technischen Fortschritt TF, sondern auch vom »Naturvermögen bzw. dem sog. „Öko-Realkapital« ÖK ab. Letzteres umfaßt die Gesamtheit der erneuerbaren und nicht-erneuerbaren Ressourcen, die vor allem bezüglich fünf Funktionen oder »Leistungen« für den Menschen und sein »künstliches « Produktionssystem von Bedeutung sind. Diese Leistungen betreffen

- die Bereitstellung von Ressourcen als Inputfaktoren für den Prozeß des »Durchflußwachstums «,

- die Aufnahme und Assimilation von Emissionen und Abfällen,

- die direkte Beeinflussung des menschlichen Wohlbefindens (z.B. Erlebniswert einer Landschaft oder Tierwelt oder Signalfunktion),

- die Biotop- oder Lebensraumfunktion für Menschen und lebende Umwelt sowie

- eine generelle Ökosystemfunktion (etwa Gewährleistung klimatischer Stabilität oder ökologischer Kreișläufe).

Unterstellt man, was die ökologische Ökonomie generell tut, eine begrenzte Substituierbarkeit des Öko-Realkapitals durch das künstliche Realkapital, das Humankapital oder den technischen Fortschritt, wird es notwendig, die entscheidende längerfristige »Entwicklungsnebenbedingung 《 oder Säule, nämlich das Öko-Realkapital, zu sichern und $\mathrm{zu}$ bewahren. Dies ist der relevante Kerngedanke des Ein-Säulen-Konzepts, das dann nur noch die Aufgabe hat, den Begriff des Öko-Realkapitals zu operationalisieren und Regeln für den Umgang mit ihm, insbesondere mit den natürlichen Ressourcen (4) (häufig als sog. Management-Regeln gekennzeichnet), abzuleiten. Diese Regeln beziehen sich vor allem auf die Nutzung nicht erneuerbarer und regenerierbarer Ressourcen und die Verhinderung kritischer Höchstbelastungen (critical loads) von Ökosystemen.

\section{Variable Leitplanken}

Die so abgeleiteten Nebenbedingungen für den Erhalt des entwicklungslimitierenden Öko-Realkapitals gleichen variablen Leitplanken, und der verbleibende Entwicklungsspielraum stellt einen variablen Entwicklungskorridor dar. Dahinter stehen eine bunte Fülle von politischen Einzelentscheidung, die in einer Art Nutzen-KostenVergleich Erkenntnisse der Wirkungsforschung sowie gesellschaftliche Wertungen oder Einstellungen (etwa Risikobereitschaft) berücksichtigen und in Einzelfällen auch zum Verbot bestimmter Stoffe führen können. Nachhaltigkeit wird hier nicht in Form eines einzuhaltenden Leitstrahls, auf dem sich die wirtschaftlichen Akteure bewe- 
gen sollen, sondern in Gestalt vielfältiger Einzelgrenzen, die den gesellschaftlichen Konsens über das, was als nicht mehr nachhaltig angesehen wird, zum Ausdruck bringen. Variabel ist dieser so abgeleitete Korridor, weil neue Erkenntnisse sowie eine gestiegene Umweltsensibilität (etwa als Folge eines gestiegenen Wohlstandes) bzw. eine steigende oder sinkende Risikobereitschaft im Zeitablauf eine Leitplankenverschiebung auslösen können.

$\mathrm{Da}$ eine solche Nachhaltigkeitskonzeption der Wirtschaft nicht vorschreibt, was sie zu tun hat, sondern nur definiert, was gesellschaftlich als nicht mehr tolerierbar angesehen wird, und gleichzeitig auf expliziten oder intuitiven NutzenKosten-Überlegungen beruht, ist sie - läßt man einmal die Instrumentenfrage außer Acht durchaus ökonomieverträglich und grundsätzlich mit der Marktwirtschaft vereinbar. Sie weist eine hohe Parallelität mit bestimmten Formen klassischer Sozialpolitik auf, die in weiten Bereichen ebenfalls nicht tolerierbare Zustände (etwa Kinderarbeit, Nachtarbeit von Frauen usw.) definierte und die Einhaltung gewisser Mindestschutzbedingungen für verbindlich erklärte. Sieht man dies als eine Politik zur Verhinderung gesellschaftlicher Spannungen und der mit ihnen verbundenen Risiken an, wurde bereits damals unter sozialpolitischer Flagge - Nachhaltigkeitspolitik betrieben.

\section{Das Drei-Säulen-Konzept: Ökologie=, Ökonomie- und Sozialverträglichkeit}

Das Drei-Säulen-Konzept geht von der Überlegung aus, daß eine befriedigende Interpretation des Nachhaltigkeitsbegriffs auch dem Tatbestand Rechnung tragen muß, daß die oben angesprochene Entwicklungsstetigkeit nicht nur durch ökologische, sondern auch durch ökonomische und soziale Risiken bedroht werden kann. So machte der Zusammenbruch sozialistischer Planwirtschaften deutlich, daß die Vernachlässigung »ökonomischer Gesetzmäßigkeiten« bzw. der Frage nach der Leistungsfähigkeit von Wirtschaftssystemen ganze Gesellschaftssysteme in Frage zu stellen vermag. Insofern verlangen die Vertreter dieses Drei-Säulen-Konzepts für eine befriedigende Definition der Nachhaltigkeitsbedingungen neben der Ökologieverträglichkeit auch die Mitberïcksichtigung ökonomischer und sozialer Verträglichkeitsaspektè.

Neben der ökologischen Nachhaltigkeits-Bedingung müssen demzufolge auch die der Ökonomie- oder Sozialverträglichkeit konkretisiert werden. Dies potenziert das Operationalisierungsproblem. Was die Ökonomieverträglichkeit betrifft, könnte man eine Minimierung der Vermeidungskosten fordern. Dies ist jedoch nicht ausreichend. So ist es nicht zulässig, allein die Kosten des Umweltschutzes zu betrachten und steigende Umweltschutzkosten als nicht zulässige »Belastung « zu charakterisieren. Sieht man nämlich die Lösung des Umweltproblems, was der ökonomischen Sichtweise entspricht, als ein Optimierungsproblem an, muß man den Kosten des Umweltschutzes die Kosten des unterlassenen Umweltschutzes bzw. den Nutzen des Umweltschutzes gegenüberstellen. Mangelnde Ökonomieverträglichkeit wäre bei einer solchen Sichtweise darum dann gegeben, wenn die Summe der Kosten des Umweltschutzes und der Kosten des unterlassenen Umweltschutzes sich nicht mehr im Minimum befänden, oder die Grenzkosten des Umweltschutzes die Grenzfolgekosten eines unterlassenen Umweltschutzes deutlich über- oder unterschreiten würden.

Aber auch hier muß man auf Probleme aufmerksam machen, die die Anwendung dieses Ansatzes zur Beurteilung der Ökonomieverträglichkeit einschränken. So sind die Schätzungen des Nutzens des Umweltschutzes oder der Kosten des unterlassenen Umweltschutzes mit großen methodischen Problemen verbunden. Dies gilt auch für die Ableitung von Nutzungsregeln von natürlichen Ressourcen. Geht man z. B. von nicht erneuerbaren Ressourcen aus, bedeuttet deren heutige Verwendung tendenziell eine Einengung des künftigen Verfügungsspielraums. Ihre Nutzung stellt darum ein intertemporales Allokations- und Verteilungsproblem dar. Je knapper der Ressourcenbestand ist, desto höher sind mit anderen Worten seine sog. Opportunitätskosten (definiert als entgangener künftiger Nutzen). Eine Entscheidung über die optimale Nutzung einer endlichen Ressource verlangt $u$. a. die Berücksichtigung

- der künftigen Nachfrage als Ausdruck von Bevölkerungsentwicklung, Wirtschaftswachstum, Einschätzung künftiger Ressourceneffizienz (Output je Ressourceneinheit) und Substituierbarkeit, Entwicklung neuer Güter und ressourcenabhängiger Úmweltbelastungen,

- eine möglichst zutreffende Einschätzung der Ressourcenreserven samt der für ihre Nutzung (Exploration, Erschließung, Förderung und Transport) notwendigen Kosten sowie

- eine Entscheidung über die Diskontrate, mit der die künftigen Nutzen und Kosten abdiskontiert werden sollen.

Letztlich impliziert die Lösung dieses zeitlichen Allokationsproblems den Vergleich gegenwärtiger und künftiger Nutzen und damit auch die Berücksichtigung der Ansprüche künftiger Generationen (intergenerationelles Verteilungsproblem). Weiter können neue und noch unbekannte Erschließungstechniken, die den Zugriff auf bislang nicht nutzbare Rohstofflagerstätten gestatten, eine mögliche Steigerung der Ressourcenproduktivität (Output je Ressourceneinheit) sowie die Schaffung von neuen Substitutionsmöglichkeiten den Handlungsspielraum künftiger Generationen erweitern, wobei diese Änderung teilweise von der heutigen Ressourcennutzung (etwa bei Ausbau der Forschungsinfrastruktur) abhängig ist. Wenn man solche Zusammenhänge unberücksichtigt läßt und die Interessen künftiger Generationen genau so hoch gewichtet wie jene der heutigen (Diskontrate von Null), gelangt man zu anderen Nutzungsregeln, als wenn man hinsichtlich der künftigen Techno- logien optimistischer ist und interessemäßig zugunsten der heutigen Generation entscheidet. Tendenziell plädiert hier die Mehrheit der Vertreter der ökologischen Ökonomie für eine Gleichgewichtung der Interessen der verschiedenen Generationen sowie einen gewissen Technologiepessimismus. Dies zeigt, daß das Ein- und das Drei-Säulen-Modell, was die Definition des Nachhaltigkeits-Korridors betrifft, sehr anfällig für normative Postulate ist und die Vertreter der ökologischen Ökonomie tendenzill niedrigere Entwicklungsspielräume unterstellen als die Vertreter der klassischen Ökonomie.

\section{Am Vorsorgeprinzip orientiertes Reduktionskonzept}

Zumeist lassen sich die Vertreter der ökologischen Ökonomie aber nicht auf eine derartige Methoden-Diskussion ein, sondern präferieren von vornherein das dritte Nachhaltigskeitskonzept, d. h. das am Vorsorgeprinzip festgemachte Reduktionskonzept. Vorsorglich unterstellt man im Interesse künftiger Generationen keinen den intergenerationellen Verteilungskonflikt mildernden technischen Fortschritt und räumt künftigen Generationen - teilweise losgelöst von der Bevölkerungsentwicklung - gleiches Nutzungsrecht an den natürlichen Ressourcen ein. Noch entscheidender ist aber, daß man in starkem Maße die Abhängigkeit des Menschen von der Natur und den mit den anthropogen induzierten Naturveränderungen verbundenen Risiken betont. Insbesondere gewichtet man die bereits oben angesprochene generelle Ökosystemfunktion hoch und fordert die ebenfalls schwer zu definierende Aufrechterhaltung der Selbstorganisations- und Selbsterhaltungskraft der Biosphäre. Unter Rückgriff auf die Ergebnisse der Chaosforschung wird vor allem auf das Risiko sprunghafter qualitativer Veränderungen der Biosphäre bei bestimmten (aber unbekannten) Belastungen verwiesen und als Vorsorgestrategie eine RisikoMinimierung eingefordert. Nach Ansicht vieler Anhänger der ökologischen Ökonomie sind die Ökosysteme zu komplex, ưm zu klaren Aussagen bezüglich der Langfristwirkungen sowie der kritischen Belastungsgrenzen zu gelangen. Im Sinne einer Pauschal-Hypothese wird jeder Stoffeintrag, und zwar losgelöst von seiner Einzelwirkung, als »belastend « sowie jede Reduktion des Stoffflußvolumens als »entlastend « und damit als risikomindernd angesehen.

Tendenziell lehnen die Vertreter der ökologischen Ökonomie und der neueren Stoffpolitik darum die oben skizzierte Korridorinterpretation $a b$ und fordern-zwecks allgemeiner Risikominderung und Berücksichtigung intergenerationaller Verteilungsgerechtigkeit - vorsorglich die Reduktion des Stoffflußvolumens bzw. die kontinuierliche Minderung der Materialintensität des Wirtschaftens. Damit bekommt die Interpretation des Nachhaltigkeitspostulats eine neue Qualität. Nicht mehr die Durchsetzung bestimmter Zustände - etwa definiert über das Unterschreiten kritischer Belastungszustände - wird zum Ziel 
erhoben, sondern der Weg, nämlich die stetige Reduktion des Stoffflußvolumens, wird als pauschale Vorsorgestrategie zum relevanten Ziel erklärt. Die Ausschöpfung eines behaupteten Reduktionsspielraums - etwa im Rahmen einer Faktor 10-, Faktor 5- oder Faktor 4-Gesellschaft wird zum Leitbild einer am Nachhaltigkeitspostulat ausgerichteten Umweltpolitik.

\section{Internalisierungsstrategie versus umfassende Lenkungsaufgabe}

Um nicht falsch verstanden zu werden: Auch die Nachhaltigkeitsinterpretationen im Sinne des ökologischen Ein-Säulen- oder des Drei-SäulenModells werden, vor allem wenn alle Länder dieser Welt die Produktions- und Konsummuster der entwickelten Industrienationen kopieren wollen, zwecks Verhinderung von Zuiständen von Nichtnachhaltigkeit mit einer Reduktion der Materialintensität, einer verstärkten Kreislaufführung der Stoffe, einer Steigerung der Langlebigkeit vieler Produkte oder mit gravierenden Änderungen der Konsummuster verbunden sein. Dies gilt aber nur solange, wie die oben angesprochenen Leitplanken, die unter ökonomischen Aspekten Grenzen darstellen, ab denen die Grenzschadenskosten die Grenzvermeidungskosten überschreiten, durchbrochen werden und die Gefahr einer nicht nachhaltigen Entwicklung besteht. Dies ist aber etwas anderes als - losgelöst von den ökonomischen Implikationen - die Überstrapazierung des Vorsorgeprinzips im Sinne des Forderns steter Reduktion des Stoffflußvolumens. Eine solche stoffpolitische Forderung nach kontinuierlicher Reduktion des Stoffflußvolumens ist auch mehr als eine Internalisierungsstrategie; sie ist eine umfassende Lenkungsaufgabe, bei der die Akteure des Wirtschaftssystems über eine sukzessiv steigende Ressourcenbesteuerung mit der staatlich geführten Preispeitsche zur Effizienz- oder Suffizienzrevolution getrieben werden sollen. Eine solche Konzeption kann man nicht mehr als ökonomieveträglich oder marktwirtschaftskonform kennzeichnen.

Dies gilt auch für jene Spielformen des Reduktionskonzepts, die als zu berücksichtigende $\mathrm{Ne}$ benbedingung die Makroneutralität - keine nachteiligen Nebenwirkungen auf Beschäftigung, Preisniveau oder wirtschaftliches Wachstum fordern (5) oder sogar behaupten, eine solche Politik sei wohlstandssteigernder Natur - eine Art makroökonomische Doppeldividende (mehr Umweltschutz mit mehr Wohlstand). Hier mischen sich normative Überlegungen - Verteilungsziele und Hochgewichtung des Vorsorgeprinzips - mit schwer nachweisbaren Behauptungen bezüglich der Makroeffekte einer Politik immer stärkerer Staatsdeterminiertheit der Ressourcenpreise, insbesondere wenn dies im natio- nalen Alleingang geschieht. Solche radikalen Forderungen bestimmter Vertreter der ökologischen Ökonomie stellen eine Transformation unseres Wirtschaftssystems dar und stehen, was ihre Makroneutralität oder ökonomische Vorteilhaftigkeit betrifft, auf schwachen Fundamenten.

\section{Paul Klemmer, Essen}

\section{Anmerkungen}

1) Vgl. Enquete-Kommission $»$ Schutz des Menschen und der Umwelt « des Deutschen Bundestages (Hrsg.): Die Industriegesellschaft gestalten. Perspektiven für ei nen nachhaltigen Umgang mit Stoff- und Materialströmen, Endbericht, Bonn 1994 (gleichzeitig als Bundestagsdrucksache 12/8260) erschienen).

2) So wurden vom Bundesminister für Wirtschaft 1995 einige Forschungsaufträge vergeben, die sich mit den ordnungspolitischen Implikationen des Nachhaltigkeitspostulàts befassen, wobei zwangsläufig auf die Forderung nach einer umfassenden Stoffpolitik eingegangen werden muß.

3) Vgl. zum nachfolgenden auch Klemmer, P.: Nachhaltige Entwicklung - aus ökonomischer Sicht, Zeitschrift für angewandte Umweltforschung, 7. Jg., 1994, S. $14 \mathrm{ff}$.

4) Vgl. etwa Enquete-Kommission »Schutz des Menschen und der Umwelt $\ll$ des Deutschen Bundestages (Hrsg.): a.a. O., S. 32

5) So z. B. die Enquete-Kommission »Schutz des Menschen und der Umwelt« des. Deutschen Bundestages (Hrsg.): a. a. O., S. 49.

\section{Monetäre Dynamik und Nachhaltigkeit}

Der Begriff der »Nachhaltigkeit « bzw. »Sustainable Development« ist seit der Veröffentlichung des Brundtland-Berichtes im Jahre 1987 zu der wahrscheinlich am häufigsten gebrauchten Vokabel in der Oekonomie geworden, wenn es darum geht, ökologische Ziele für die Zukunft zu formulieren. In diesem Zusammenhang hat man sich bis heute aber nur wenig mit der längerfristigen Dynamik heutiger Wirtschaftssysteme beschäftigt. Es wird kaum danach gefragt, welche Logik hinter den wirtschaftlichen Vorgängen steckt und welche Konsequenzen sich daraus für Wirtschaft und Umwelt ergeben. Erst wenn man sich aber mit diesen Themen beschäftigt wird man auch Antworten auf nicht ganz unerhebliche Fragen finden, wie

- Besteht im heutigen Weltwirtschaftssystem ein Zwang zum Wachstum oder ist auch eine quantitativ nicht mehr wachsende Wirtschaft möglich?

- Lässt sich Wirtschaftswachstum mit nachhaltiger Entwicklung vereinbaren, d. h., kann es ein nachhaltiges Wachstum überhaupt geben? Im folgenden versucht dieser Beitrag, zumindest einige Aspekte der wirtschaftlichen Wachstumsdynamik zu beschreiben, um so einer Antwort auf die eben gestellten Fragen näherzukommen.

\section{Monetäre Dynamik und Wachstumszwang}

Im Grunde genommen geht es in einer Geldwirtschaft, so wie sie in den heutigen Industrieländern schon seit hunderten von Jahren existieren, nur um eines: nämlich aus einer bestimmten Geldsumme M (money) eine höhere Geldsumme M' zu machen. Dies ist der entscheidende Grund, weshalb ein Unternehmen überhaupt produziert. Unternehmen produzieren, weil sie damit rechnen, durch den Verkauf von Gütern bzw. Dienstleistungen im Verlauf der Zeit eine höhere Geldmenge $M$ ' einzunehmen als sie ursprünglich für die Bezahlung der Produktionsfaktoren Arbeit (Löhne) und Kapital (Investitionen) ausgegeben haben, um so einen Gewinn $G=M^{\prime}-M$ zu reali sieren (1). Dies ist, ganz einfach ausgedrückt, die fundamentale Logik einer Geldwirtschaft, welche diese zu einer ständigen Expansion antreibt (2).

Der wirtschaftliche Kreislauf ist somit für das Unternehmen ein kontinuierlicher Prozess, der sich in einer auf Marx (und nicht auf den Marxismus!) zurückgehenden Terminologie als M-CM'-Kreislauf (Geld-Ware-Geld-Kreislauf) beschreiben lässt. Der Kreislauf beginnt damit, dass ein Unternehmen eine bestimmte Geldsumme M investiert, um damit die für die Produktion notwendigen Produktionsfaktoren Arbeit und Kapital zu bezahlen. Erst an zweiter Stelle kommen reale, d. h. physische Güter C (commodities) hin$\mathrm{zu}$, indem die Unternehmen Anlagen und Maschinen kaufen (Realkapital), um damit Güter und Dienstleistungen zu produzieren. Durch den Verkauf der mit Hilfe von Arbeit und Kapital hergestellten Güter verwandelt das Unternehmen schliesslich die produzierten Güter und Dienstleistungen wieder in Geld (M'), wobei gilt, dass die erwartete Geldmenge M' grösser sein muss als die ursprünglich investierte Geldmenge $\mathrm{M}$. Die in dem Wirtschaftskreislauf zirkulierende Geldmenge vergrössert sich somit von Periode zu Periode, solange sich die Gewinnerwartungen tatsächlich erfüllen.

Handeln alle Unternehmen in einer Wirtschaft nach dem Prinzip des M-C-M'-Kreislaufs, so wächst auch die gesamte Wirtschaft, sofern die erwarteten Einnahmen M' sich bei einer Mehrheit der Unternehmen auch tatsächlich einstellen. Solange der M-C-M'-Kreislauf funktioniert, funktioniert auch die Wirtschaft. Dank des Wachstums ist die Wirtschaft kein Nullsummenspiel, sondern ermöglicht es den Wirtschaftsakteuren, einen Gewinn zu machen bzw. das Einkommen zu erhöhen, ohne dass sich dadurch der Gewinn bei andern Wirtschaftsakteuren bzw. das Einkommen verringern muss, wie dies bei einem Nullsummenspiel der Fall wäre. Das ist ein we- 
(c) 20I0 Authors; licensee IÖW and oekom verlag. This is an article distributed under the terms of the Creative Commons Attribution Non-Commercial No Derivates License (http://creativecommons.org/licenses/by-nc-nd/3.o/), which permits unrestricted use, distribution, and reproduction in any medium, provided the original work is properly cited. 